\title{
SISTEMAS DE PROGRAMAÇÃO DA PRODUÇÃO COM CAPACIDADE FINITA: UMA DECISÃO ESTRATÉGICA?
}

Marcelo Caldeira Pedroso
Mestre e Engenheiro de Produção pela Escola

Politécnica/USP. Consultor da Gianese Corrêa \&

Associados

Henrique Luiz Corrêa PhD em Operations Management pela Universidade de Warwick, Inglaterra. Professor do Departamento de Engenharia de Produção da Escola Politécnica/ USP. Consultor da Gianese Corrrêa \& Associados.

RESUMO: Este artigo analisa o impacto, na competitividade das empresas, da crescente utilização em nivel nacional e mundial, dos denominados "sistemas de programaçāo da produçāo com capacidade finita". Para tanto, o artigo conceitua estes sistemas, bem como discute as possiveis implicações decorrentes da implantação destes sistemas na estratégia de manufatura das empresas. Os autores propõem uma classificação destes sistemas e apresentam brevemente aqueles comercialmente disponiveis no Brasil.

ABSTRACT: This paper examines the impact of the increasingly growing use of the so called "finite capacity scheduling systems" on companies competitiveness. With this aim, the article defines relevant concepts regarding finite scheduling systems and discusses the implications of adopting/implementing them for the adopter's manufacturing strategy. The authors also propose a taxonomy for finite scheduling systems and survey the current finite scheduling software applications available in Brazil.

PALAVRAS-CHAVE: programação da produção com capacidade finita, planejamento e controle da produçāo, competitividade, estratégia de manufatura.

KEY-WORDS: finite scheduling, production planning and control, competitiveness, manufacturing strategy. 
A busca da competitividade por parte das empresas, notadamente quando se objetiva reduzir os custos - associados aos estoques e ao nível de utilização e variação da capacidade produtiva - e melhorar $o$ nível de serviço percebido pelo cliente em termos de uma maior velocidade de entrega, uma melhor pontualidade nos prazos acordados e um aumento de flexibilidade em relação às variações da demanda e dos recursos produtivos - coloca o sistema de planejamento, programaçãoe controle da produção (PPCP) como uma área de decisão prioritária para os executivos nos $\operatorname{anos} 90$.

Basicamente, o sistema de PPCP é uma área de decisão da empresa que objetiva planejar e controlar os recursos alocados ao processo produtivo visando atender a demanda dos clientes.

Tradicionalmente, a literatura tem abordado predominantemente sistemas como MRPII, JIT e OPT, que já há alguns anos fazem parte do jargão da área. Recentemente, um novo conjunto de sistemas e termos como Leitstand, finite capacity scheduling systems, simuladores, manufacturing execution systems e manufacturing operation management systems têm sido crescentemente divulgados na literatura. ${ }^{2}$ Todos estes sistemas são, basicamente, centrados na utilização de aplicativos informatizados como ferramenta de suporte às decisões em administração da produção e, particularmente, em PPCP.

O mercado apresenta hoje centenas de alternativas de aplicativos de software para apoiar a implantação e as decisões decorrentes destes sistemas. Entre estes aplicativos estão aqueles que utilizam a lógica MRPII ${ }^{3}$ - que apresentam todos, em linhas gerais, uma mesma estrutura básica de solução do problema ${ }^{4}$ e variam entre si nas funcionalidades que cada particular aplicativo de software apresenta. Além dos aplicativos que se utilizam da lógica MRPII, outras soluções mais específicas e ainda em estágio menos avançado de desenvolvimento têm sido recentemente disponibilizados comercialmente: os sistemas de programação da produção com capacidade finita (ou finite capacity scheduling sistems, cujo conceito será apresentado mais adiante neste artigo), que possuem cada um sua lógica própria de solução de problema - normalmente baseada em simulação em computador - , sem que ainda se tenha chegado a um design básico que, como ocorre com o MRPII, domine a abordagem adotada pelos diversos fornecedores de sistemas.

A decisão sobre a adoção de um sistema de PPCP deve considerar a multiplicidade de soluções hoje possiveis, assim como a adequação destas ao ambiente particular de cada empresa. Na realidade, entretanto, as decisões envolvendo a escolha e implantação de sistemas de PPCP têm ocorrido nas empresas, em muitos casos, segundo uma lógica que pode ser caracterizada como induzidas por promessas de panacéias, em detrimento da escolha de um sistema que melhor suporte a estratégia competitiva da empresa. Podem-se verificar casos em que foram investidos alguns milhões de dólares no processo de desenvolvimento (que pode envolver a escolha de alternativas de pacotes de software) e implantação de sistemas de PPCP, sendo que os resultados esperados jamais tenham sido alcançados. ${ }^{5}$ Este fato decorre, dentre outros possíveis fatores, da falta de uma metodologia adequada que suporte o projeto, escolha e implantação de um sistema de PPCP que seja adequado às necessidades estratégicas da empresa, e que considere sistemicamente suas competências e restrições. ${ }^{6}$

Este artigo objetiva analisar o impacto, na estratégia de manufatura das empresas, da adoção de determinado sistema de PPCP. Em particular, serão enfatizados os denominados sistemas de programação da produção com capacidade finita, já que o desenvolvimento rápido da área não tem sido acompanhado por discussões da literatura, particularmente, sobre a gestão e a adequação destes sistemas às necessidades estratégicas específicas de cada empresa, um aspecto essencial a ser considerado na decisão de implantá-los.

\section{O IMPACTO ESTRATÉGICO DAS DECISÕES RESULTANTES DE UM SISTEMA DE PPCP}

Os sistemas de PPCP objetivam apoiar as decisões de o que, quanto, quando e onde produzir e o que, quanto e quando comprar. Estas decisões definem quatro determinantes fundamentais do desempenho destes sistemas:
1. Veja, por exemplo, NEWMAN, W. E SRIDHARAN, V. Manufacturing planning and control: is there one definitive answer? Production and inventory management journal, First Quarter, p. 50-54, 1992 e CORREA, H.L. \& GIANESI, I.G.N. Just inTime, MRPII e OPT: um enfoque estratégico. São Paulo: Atlas, 1993.

2. Para maiores detalhes veja ADELSBERGER, H. H. e KANET, J.J. The Leitstand: A New Tool for Computer-Integrated Manufacturing. Production and Inventory Management Joumal, $v$. 32, n. 1, p. 43-48, 1991; HAKANSON, W. P. Managing Manufacturing Operations in 1990's. Industrial Engineering, p. 31-34, julho, 1994; HARRISON, M. Finite Capacity Moves to the Heart of MRPII. Manufacturing Systems, p.12-16, May 1994; HLUPIC, V. e PAUL, R. J. A Critical Evaluation of Four Manufacturing Simulators. International Journal of Production Research, v. 33, n. 10, p. 2757-2766, 1995; PORTER, J. K., JARVIS, P., LITTLE, D., LAARKMANN, J., HANEN, C. e SCHOTTEN, M. Production Planning and Control System Developments in Germany. International Journal of Operations \& Production Management, v. 16, n. 1, p. 2739, 1996

3 Baseado no cálculo de necessidades de recursos (de que tipos, em que quantidades e em que datas) para 0 atendimento a necessidades de produçăo de produtos finais de forma a permitir cumprimento de prazos e mínima formação de estoques. Veja CORREA, H.L. E GIANESI, I.G.N. Op. cit para uma descriçáo mais detalhada de sistemas MRPII.

4. Esses sistemas já convergiram para um design dominante, segundo conceito proposto por UTTERBACH, D. Mastering the Dinamics of Innovation. Boston: Harward Business School Press, 1994.

5. Segundo BERRY, W. L. e HILL, T. Linking Systems to Strategy. International Journal of Operations \& Production Management, v. 12, n.10, p. 3-15, 1992.

6. Veja PEDROSO, M. C. MISPEM: Modelo de Integração do Sistema de PPCP à Estratégia de Manufatura. Dissertação de Mestrado, Escola Politécnica da Universidade de São Paulo, São Paulo, 1996, para um tratamento detalhado sobre o tema. 


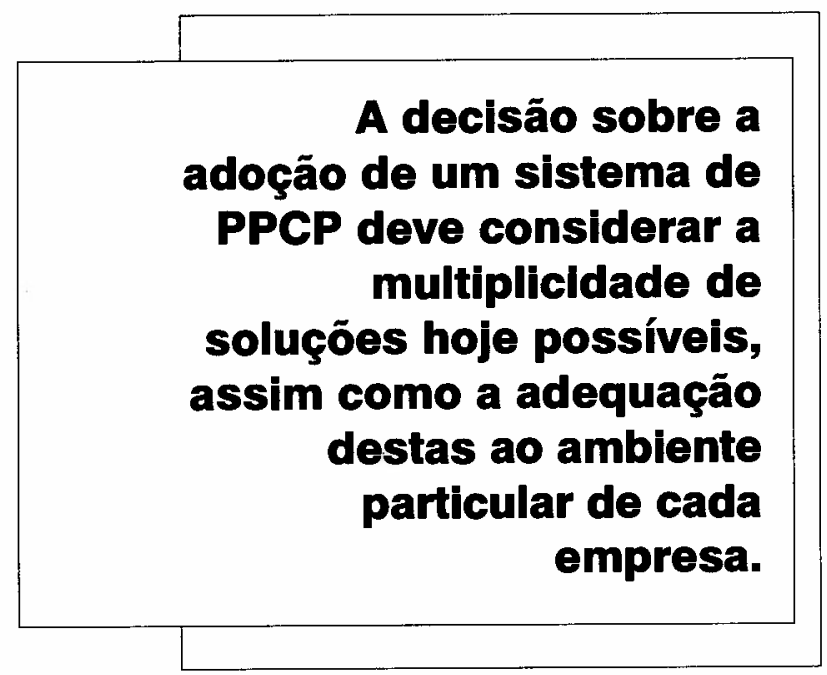

- os níveis, em volume e mix, de estoques de matérias-primas, produtos em processo e produtos acabados;

- os níveis de utilização e de variação da capacidade produtiva (e, conseqüentemente, os custos financeiros e organizacionais decorrentes de ociosidade, hora extra, demissão, contratação, subcontratação e outros);

- o nível de atendimento à demanda dos clientes, considerando a disponibilidade dos produtos em termos de quantidades e prazos de entrega;

- a competência quanto à reprogramação da produção, abordando a forma como a empresa reage às mudanças não previstas nos seus recursos de produção $e$ na demanda dos clientes.

Estes determinantes podem ser desdobrados em objetivos de desempenho específicos para o Sistema de PPCP . Estes objetivos - apresentados a seguir - podem ter maior ou menor importância relativa conforme a situação competitiva específica tratada, bem como apresentar relações de desempenho negativas ou positivas - ou seja, gestões para a melhoria no desempenho de determinado desempenho repercutem negativamente ou positivamente no desempenho do outro:

- manter o nível mínimo desejável de estoque de matérias-primas;

- manter o nível mínimo desejável de estoque de produtos em processo;
- manter o nível mínimo desejável de estoque de produtos acabados;

- atingir o nível adequado de utilização da capacidade produtiva;

- manter um nível adequado de variação da capacidade produtiva;

- atingir o nível adequado de atendimento à demanda; $\mathrm{e}$

- reprogramar a produção na ocorrência de mudanças não previstas nos recursos produtivos ou na demanda, considerando o timing adequado e a consistência em relação aos demais objetivos de desempenho do sistema.

O desempenho do sistema de PPCP, por outro lado, impacta diretamente o desempenho da manufatura, caracterizado pelos seguintes objetivos de desempenho:

- custo, que diz respeito à capacidade de a empresa fabricar produtos com alta eficiência na utilização dos recursos produtivos;

- qualidade, que se refere a fazer produtos de acordo com as especificações (qualidade no processo) e que atendam às necessidades e expectativas dos clientes (qualidade no projeto);

- velocidade, que está relacionado à habilidade da empresa em entregar produtos mais rapidamente do que a concorrência;

- pontualidade, que representa a capacidade de a empresa cumprir os prazos de entrega prometidos ao cliente; $\mathrm{e}$

- flexibilidade, que se conceitua como a habilidade de a manufatura adapatar-se com eficácia e eficiência às mudanças não planejadas nos seus ambientes interno e externo.

O desempenho interno da manufatura, por sua vez, condiciona o desempenho externo da empresa - aquele percebido pelo cliente.'

\section{O CONCEITO DE SISTEMA DE PROGRAMAÇÃO DA PRODUÇÃO COM CAPACIDADE FINITA}

As decisões do sistema de PPCP ocorrem em diferentes horizontes de tempo e períodos de replanejamento, bem como consideram diferentes níveis de agregação 
da informação. Estas decisões podem ser classificadas em três níveis: planejamento de longo, médio e curto prazo e controle. Este conceito está relacionado ao denominado planejamento hierárquico da produção, uma metodologia que propõe decompor o problema do planejamento da produção de larga escala em sub-problemas menores, resolvendo-os seqüencialmente - do maior horizonte de tempo para o menor $-\mathrm{e}$ iterativamente - as decisões nas hierarquias superiores são restrições aos problemas seguintes, bem como são realimentadas por estes.

Desta forma, as decisões relacionadas aos três níveis e à função controle estão intrinsecamente interrelacionadas, o que implica que um sistema de PPCP deve ser projetado considerando este conjunto de decisões, bem como a importância relativa de cada nível de decisão dentro do contexto particular da cada empresa.

A programação da produção aborda o planejamento de curto prazo. Basicamente, a programação da produção consiste em decidir quais atividades produtivas (ou ordens de trabalho) devem ser realizadas, quando (momento de início ou prioridade na fila) e com quais recursos (matérias-primas, máquinas, operadores, ferramentas, entre outros) para atender à demanda, informada ou através das decisões do plano mestre de produção ou diretamente da carteira de pedidos dos clientes (Figura 1). Este conjunto de decisões é dos mais complexos dentro da área de administração da produção.

Isto se deve principalmente ao volume de diferentes variáveis envolvidas e sua capacidade de influenciar os diferentes e conflitantes objetivos de desempenho do Sistema de PPCP. Assim, as decisões decorrentes da programação da produção se tornam um problema combinatório de tal ordem que soluções intuitivas são inadequadas pelas limitações humanas de administrar informações.

Para exemplificar, pode-se citar algumas das diversas possibilidades e restrições:

Em termos de ordens:

- as ordens, geralmente, apresentam datas de entrega diferentes;

\section{FIGURA 1. ESQUEMA SIMPLIFICADO dAS VARIÁVEIS DE DECISÃo EM PROGRAMAÇÃO DA PRODUÇÃO}

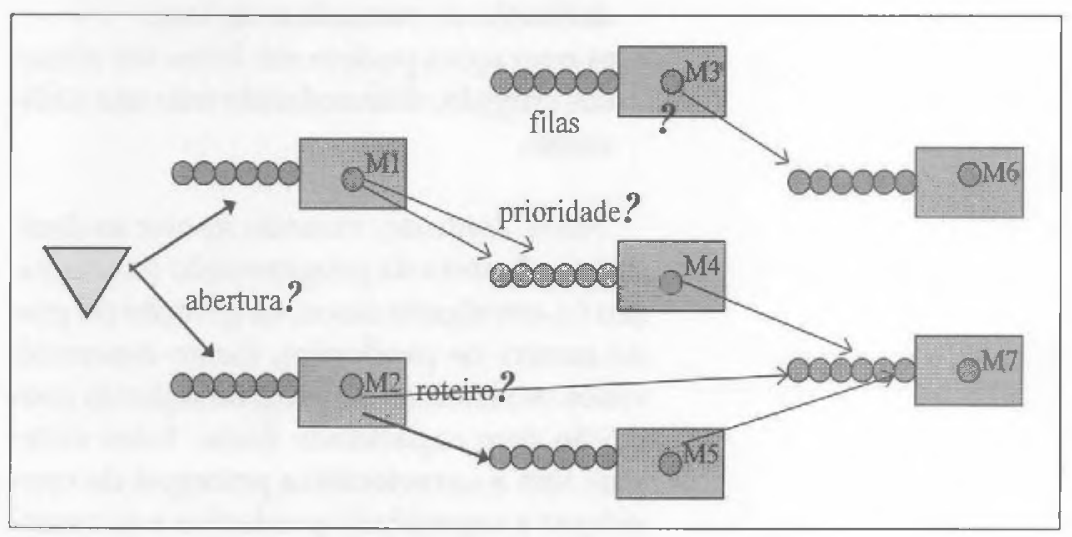

- cada ordem, geralmente, está em um estado diferente de completude;

- as ordens podem apresentar set up ${ }^{11}$ com tempos e atividades variáveis, em função da ordem anterior;

- cada ordem pode ter roteiros alternativos, dependendo das características tecnológicas dos equipamentos;

- cada ordem pode eventualmente ser feita em máquinas alternativas com eficiências diferentes;

- cada ordem pode ser de clientes com importância relativa diferente;

- cada ordcm pode necessitar de reprogramações frequentes, tanto em função dos clientes (alterações nas quantidades e nos prazos de entrega) quanto de ocorrências não previstas quanto aos recursos ou às operações.

Em termos de recursos:

- as máquinas geralmente quebram, bem como demandam manutenção;

- as matérias-primas podem não estar disponíveis;

- as ferramentas podem não estar disponíveis;

- os funcionários podem faltar.

Em termos de operações:

- os problemas relacionados à qualidade geralmente ocorrem, requerendo retrabalhos;

- as operações podem ter tempos de perecibilidade;
10. Para maiores detalhes sobre sistemas de PPCP veja VOLLMANN, T. O., BERRY, W. L. \& WHYBARK, D. C. Manufacturing Planning and Control Systems. Illinois: Richard D. Irwin, Inc., 1992

11. Preparação para início da produçẩo. 
- as operações podem demandar tempo de pós-produção (cura, secagem etc.);

- as operações podem ter restrições para a definição de tamanhos de lote;

- as operações podem ser feitas em recursos gargalo, demandando máxima utilização.

Neste contexto, visando apoiar as decisões no âmbito da programação da produção (e, em alguns casos, na geração do plano mestre de produção), foram desenvolvidos os sistemas de programação da produção com capacidade finita. Estes sistemas têm a característica principal de considerar a capacidade produtiva e as características tecnológicas do sistema produtivo como uma restrição a priori para a tomada de decisão, buscando garantir que o programa de produção resultante seja viável. Nestes sistemas, o usuário:

- modela o sistema produtivo - por exemplo: máquinas, mão-de-obra, ferramentas, calendário, turnos de trabalho, e informa os roteiros de fabricação, as velo- cidades de operação, as restrições tecnológicas, os tempos de setup e a respectiva matriz de dependência;

- informa a demanda - determinada pelo plano mestre de produção, pela carteira de pedidos ou por previsăo de vendas, bem como as alterações ocorridas - por exemplo, mudanças nas quantidades ou nos prazos de entrega;

- informa as condições reais do sistema produtivo - por exemplo, matéria-prima disponível, quebra de máquinas, manutenções; e

- modela alguns parâmetros para a tomada de decisões - por exemplo, define algumas regras de liberação (dispatching rules) ou pondera determinados objetivos a serem atingidos;

de modo que o programa de produção resultante atenda as condições particulares do sistema produtivo modelado, ou seja, dopisode-fábrica e busque maximizar os múltiplos e conflitantes objetivos de desempenho doSistema de PPCP. A figura 2 representa o ambiente em que este sistema opera.

\section{Figura 2. REPRESENTAÇÃo dA OPERAÇÃo dE UM SISTEMA DE PROGRAMAÇÃo DA PRODUÇĀO COM CAPACIDADE FINITA.}

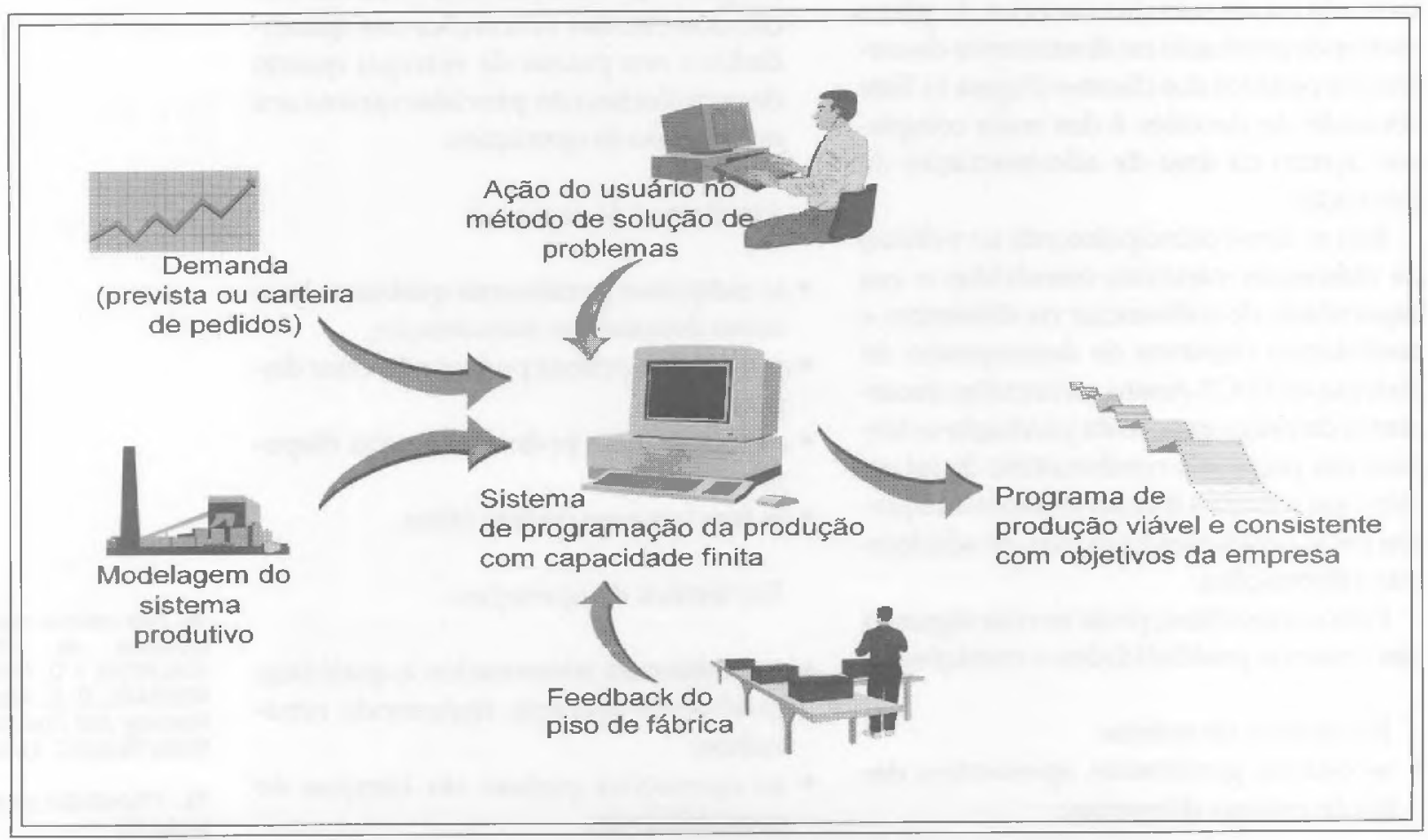


Alguns dos principais fatores que podem ser apontados como impulsionadores do desenvolvimento destes sistemas são:

- a busca de competitividade pelas empresas, aliada ao melhor entendimento das implicações das decisões resultantes do sistema de PPCP e, particularmente, da programação da produção, na estratégia de manufatura das empresas;

- as limitações dos Sistemas MRP-II em gerar programas de produção viáveis pelo fato de utilizar o conceito de capacidade infinita, ou seja, não considerar as características tecnológicas de sistema produtivo e a capacidade produtiva como limitações para a programação da produção, apenas apontando as inconsistências em termos de utilização da capacidade a posteriori - e do Kanban, quando aplicado em ambientes altamente dinâmicos e com demanda variável;

- o desenvolvimento das técnicas de simulação e de algoritmos baseados em inteligência artificial (tais como beam search, simulated annealing, genetic algorithm e tabu search), disponibilizados como ferramentas práticas aplicadas ao problema da programação da produção;

- o crescente desenvolvimento dos equipamentos (hardware), que passaram a permitir a resolução do problema da programação da produção a partir das técnicas acima citadas em tempos viáveis na prática, fato impossível no passado devido ao grande volume de informações que necessitam ser processadas.

\section{O IMPACTO RESULTANTE DA IMPLANTAÇÃO DE UM SISTEMA DE PROGRAMAÇÃO DA PRODUÇĀO COM CAPACIDADE FINITA}

O resultado da implantação de um sistema de programação da produção com capacidade finita está intrinsicamente relacionado a três fatores fundamentais:

a) adequação atual e futura deste sistema em relação ao ambiente da empresa, que envolve, entre outros:

- a efetiva necessidade da empresa em gerenciar sua capacidade de forma detalhada. Há casos em que a capacidade produtiva pode não ser um fator restritivo para a gestão da empresa no curto prazo; pode-se citar como exemplo as empresas que atuam com excesso de capacidade para atender os clientes assim que estes solicitarem - bastando, para tanto, a utilização sistemática da regra FIFO;

- o alinhamento à política de planejamento da produção da empresa. Basicamente, estes sistemas são importantes para apoiar a geração de programas de produção às empresas que utilizam a política de produção sob encomenda e programas de montagem naquelas que realizam a montagem final sob encomenda, principalmente naquelas empresas onde a demanda é pouco previsível. Por outro lado, estes sistemas também podem apoiar a definição do plano mestre de produção, que é de fundamental importância na definição dos níveis de estoque de produtos acabados (em empresas que fabricam para estoque) e dos níveis de semi-acabados (nos casos de empresas que utilizam a política de montagem sob encomenda), particularmente em ambientes onde a demanda é variável;

- a complexidade das decisões no âmbito da programação da produção. Deve-se considerar que algumas empresas "criam" a necessidade de decisões complexas para a programação da produção, seja, por exemplo, por falta de foco ou

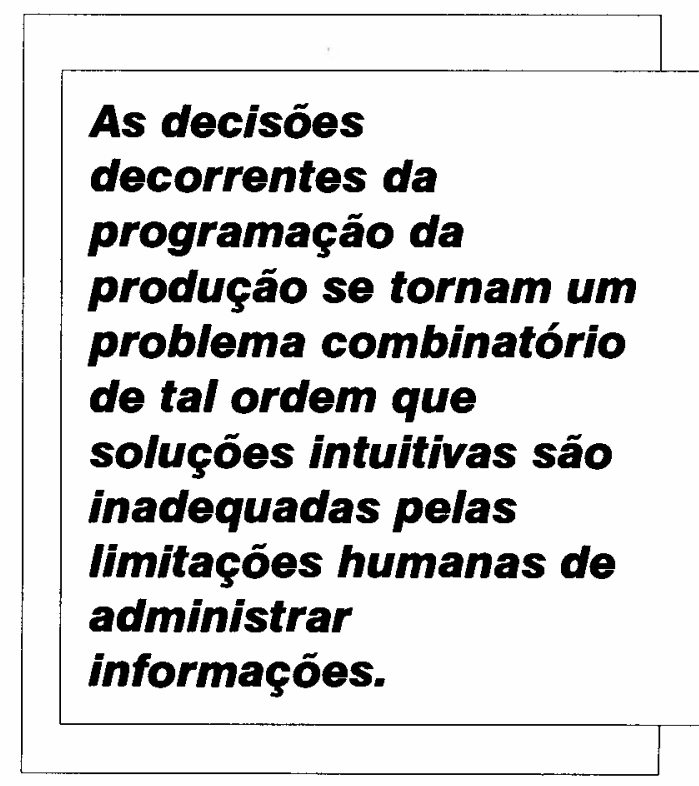

12. Para maiores detalhes sobre a metodologia de resolução dos problemas da programação da produção veja MORTON, T. E. e PENTICO, D. W. Heuristic Scheduling Systems. New York: John Wiley \& Sons, Inc.,. 1993.

13. First in, first out, ou "o primeiro a chegar é o primeiro a sair". 
Os sistemas de programação da produção com capaciadade finita, conforme explicitado anteriormente, não apresentam um design básico predominante, uma vez que existem conceitos diferentes na concepção e no escopo das decisóes apoladas por estes.

por apresentar um processo produtivo não-estável; assim, cabe avaliar se o sistema deve ser realmente implantado para gerenciar esta complexidade ou se a operação pode ser simplificada.

b) escolha de um sistema que atenda as necessidades e particularidades da empresa, que compreeende:

- a definição conceitual do sistema, considerando a adequação à empresa e determinando as decisões a serem apoiadas pelo sistema - por exemplo: geração do plano mestre de produção, dos programas de produção e de montagem, gestão das matérias-primas e do ferramental - e a integração destas decisões ao sistema de PPCP da empresa;

- a escolha dentre os diversos sistema disponíveis no mercado (ou, eventualmente, do desenvolvimento de um específico para a empresa), abrangendo o alinhamento deste em relação ao sistema definido conceitualmente, a verificação da forma como o sistema aborda as particularidades relevantes da empresa - por exemplo: roteiros alternativos em máquinas com velocidades e seqüências de operação diferentes, tempos de set up dependentes da operação anterior, mãode-obra com diferentes graus de qualificação e experiência -, a avaliação do grau de capacitação e confiabilidade do fornecedor deste, bem como a consideração de eventuais restrições internas (por exemplo: nível de investimento disponível, as especificações de hardware e de software previamente adotadas pela empresa).

c) metodologia de implantação, que deve considerar principalmente:

- o envolvimento dos recursos humanos, considerando o comprometimento da alta administração e a participação das pessoas corretas nas etapas necessárias de todas as fases de implantação do projeto;

- o treinamento, que envolve a definição das necessidades de treinamento - tanto conceitual quanto prático - e a efetivação adequada deste;

- a gestão do projeto, abrangendo o dimensionamento dos recursos, o cronograma, o controle e, eventualmente, a reprogramação das fases de implantação.

Assim, a correta definição conceitual, a escolha dentre os sistemas disponíveis e efetiva implantação de um sistema de programação da produção com capacidade finita, integrada às demais decisões do sistema de PPCP, pode capacitar a empresa a melhorar o desempenho em:

- custos: relacionados à utilização e variação da capacidade produtiva - o que pode resultar em um aumento da capacidade produtiva disponivel -, e à manutenção dos níveis de estoques de matérias-primas, em processo e de produtos acabados, adequados à política de planejamento da produção da empresa;

- velocidade de entrega: que diz respeito à redução dos tempos de atravessamento e consequentemente, à diminuição dos tempos de entrega percebidos pelos clientes;

- pontualidade nos prazos de entrega acordados: referentes à melhoria da definição dos prazos junto aos clientes (ao menos, considerando a negociação sob a ótica da programação da produção) e cumprimento destes;

- flexibilidade de volume e de entrega: que está relacionada ao aumento da habilidade da empresa em se adaptar às 
mudanças não-previstas na demanda e nos recursos produtivos, bem como a não contribuir na deterioração do desempenho em qualidade do produto em processos não-estáveis.

Por outro lado, a implantação destes sistemas exige investimentos em:

- software: que diz respeito ao aplicativo propriamente dito, às eventuais necessidades de adaptaçāo deste e à sua integração aos demais sistemas informatizados da empresa;

- hardware: que abrange os equipamentos necessários à gestão do sistema (incluindo, por exemplo, a necessidade de equipamentos dedicados e de outros para acesso e informação, além do sistema de coleta de dados) e a interligação física entre eles, e entre estes e os demais equipamentos da empresa;

- treinamento: que está relacionado à capacitação dos recursos humanos para a gestăo da nova tecnologia, incluindo a capacitação para operar o sistema - o conhecimento das funcionalidades do software - e, talvez mais importante, o treinamento conceitual, ou seja, a capacitação na gestão à qual o sistema suporta;
- implantação: que diz respeito ao processo de start up do sistema, abrangendo a modelagem deste - a representação do sistema produtivo (por exemplo, os roteiros de fabricação dos produtos, as características das máquinas, da mão-deobra e das ferramentas) e do processo de tomada de decisão (como, por exemplo, a definação das regras de liberação ou a ponderação de objetivos a serem atingidos) - e a disponibilização das demais informações necessárias (por exemplo, as matérias-primas disponiveis, os status das ordens abertas e informações sobre a demanda);

- manutenção do sistema: que considera os valores associados à gestão do sistema e à manutenção e atualização do software e do hardware;

- mudanças organizacionais: que aborda as mudanças na organização necessárias à efetiva gestão do sistema (como, por exemplo, alterações na estrutura organizacional, implantação de novos procedimentos e métodos ou contratação de recursos humanos com a qualificação adequada); que, somados, chegam a níveis relativamente elevados. ${ }^{15}$ A figura 3 ilustra os critérios de avaliação desta decisão.

\section{Figura 3. CRItÉrios de decisão para a IMPLANTAÇÃo de UM SISTEMA DE PROGRAMAÇÃO DA PRODUÇÃO COM CAPACIDADE FINITA}

\begin{tabular}{|c|c|c|}
\hline $\begin{array}{c}\text { NIVEIS DE INVESTIMENTO } \\
\text { E ESFORCOS } \\
\text { ORCANIZACIONAIS } \\
\text { EXIGIDOS }\end{array}$ & $\begin{array}{c}\text { MELHORIA } \\
\text { DE DESEMPENHO } \\
\text { DA MANUFATURA }\end{array}$ & $\begin{array}{l}\text { ANÁLISE DA } \\
\text { ADEQUAÇÃO ATUALE } \\
\text { FUTURA DO SISTEMA }\end{array}$ \\
\hline $\begin{array}{l}\text { - software } \\
\text { - hardware } \\
\text { - treinamento } \\
\text { - implantação } \\
\text { - manutenção do sistema } \\
\text { - mudanças organizacionais }\end{array}$ & $\begin{array}{l}\text { reduçáo em custos decor- } \\
\text { rentes da melhor gestáo dos } \\
\text { estoques e da capacidade } \\
\text { produtiva } \\
\text { - aumento da velocidade de } \\
\text { entrega } \\
\text { - melhoria na pontualidade de } \\
\text { entrega } \\
\text { - aumento da flexibilidade de } \\
\text { volume e de entrega }\end{array}$ & $\begin{array}{l}\text { - necessidade de gestáo } \\
\text { da capacidade } \\
\text { - alimhamento à política de } \\
\text { planejamento da produ- } \\
\text { ção } \\
\text { - complexidade das deci- } \\
\text { sóes } \\
\text { - funcionalidades e escopo } \\
\text { das decisōes dos siste- } \\
\text { mas disponíveis }\end{array}$ \\
\hline
\end{tabular}

14. Segundo COSTA, R.S. Pontualidade Total. Tese de Doutorado, Universidade Federal do Rio de Janeiro, Rio de Janeiro, 1996.

15. O principal fator financeiro, na maioria dos casos, está relacionado ao custo do software. Dentro de um universo de 14 sistemas pesquisados e disponiveis no Brasil no final de 1995 - citados adiante -, os valores variaram dentro de uma faixa de 7 mil a 1,5 milhão de dólares. 
16. Baseado em MORTON, T. E. e PENTICO, D. W. Op. cit.

17. Panwalker, S. S. e Iskander, W. A Survey of Scheduling Rules Operations Research, v. 25, n. 1 p. 45-61, Jan.-Febr. 1977.

18. PARUNAK, H. V. D. Caracterizing the Manufacturing Scheduling Problem. Journal of Manufacturing Systems, v. 10, n. 3, p. 241-258, 1991.

19. Para maiores detalhes veja por exemplo, GOLDRATT, E. M. Computerized Shop Floor Scheduling. International Journal of Production Research, v. 26, n. 3, p. 443-455, 1988; Haystack Syndrome. New York: North River Press, 1990; CORRÊA, H.L. E GIANESI, I.G.N Op. cit.

20. Como exemplos de aplicação veja OW, P. S. e MORTON, T. E. Filtered Beam Search in Scheduling. Internationa/ Journal of Production Research, v. 26, n.1, p.35-62, 1988; STORER, $H$. S., WU, S. D. e VACCARI, R. New Search Spaces for Sequencing Problems with Applications to Job Shop Scheduling Management Science, v. 38 , ח.. 10, p. 1495 1509, October 1992; ITOH, K. HUANG, D eENKAWA, T Twofold Look-Ahead Search for MultiCriterion Job Shop Scheduling. International Journal of Production Research, v. 31, n. 9 p. 2215-2234, 1993; BARNES, J. W. e CHAMBERS, J. B. Solving the Job Shop Scheduling Problem with Tabu Search. IIE Transactions, n. 25, p. 257-263, 1995.
Em resumo, a decisão de implantar um sistema de programação da produção com capacidade finita envolve a análise:

- do impacto estratégico resultante, considerando a melhoria do desempenho da manufatura nos objetivos desempenho relevantes para a competitividade da empresa;

- dos níveis de investimento e esforços organizacionais exigidos;

- dos sistemas disponíveis que, por apresentarem sensíveis diferenças tanto na concepção quanto no escopo da decisão, merecem uma avaliação mais criteriosa quanto à adequação às necessidades atuais e futuras da empresa.

\section{UMA PROPOSTA DE CLASSIFICAÇÃO DOS SISTEMAS DE PROGRAMAÇÃO DA PRODUÇÃO COM CAPACIDADE FINITA}

Os sistemas de programação da produção com capaciadade finita, conforme explicitado anteriormente, não apresentam um design básico predominante, uma vez que existem conceitos diferentes na concepção e no escopo das decisões apoiadas por estes. Pode-se, entretanto propor a seguinte classificação, baseada em três critérios básicos:

a) método de solução do problema, ou seja, de acordo com a abordagem utilizada para gerar o programa de produção: ${ }^{16}$

- sistemas baseados em regras de liberação: estes sistemas utilizam regras que decidem qual ordem, dentre uma fila de ordens disputando um recurso, será processada primeiro. A literatura apresenta cerca de uma centena destas regras ${ }^{17}$ algumas das mais conhecidas são: SPT (shortest operation process time onde ganham prioridade as ordens de menor tempo de operação) e EDD (earliest job due date, onde ganham prioridade as operações dos trabalhos que estão prometidos para as datas mais próximas). Os sistemas baseados em regras de liberação adotam o conceito de programação finita para frente, onde a capacidade do recurso vai sendo preenchida seqüencialmente em função da decisão de libera- ção das ordens. Estes sistemas são os mais difundidos atualmente dada a relativa simplicidade de modelagem e rapidez de processamento; por outro lado, exigem critérios na escolha das regras - geralmente determinadas a partir de simulação e comparação dos resultados segundo parâmetros mensuráveis, bem como pode, dependendo da escolha da regra e do ambiente em que este sistema é implantado, gerar resultados relativamente 'pobres'.

- sistemas matemáticos otimizantes: a característica principal destes sistemas reside na utilização de algoritmos matemáticos otimizantes, ou seja, o resultado da decisão é o melhor possível uma vez definido o objetivo que se deseja atingir. As soluções típicas destes sistemas são aquelas que utilizam algoritmos da pesquisa operacional. Na prática, os sistemas matemáticos otimizantes estão limitados a problemas restritos-basicamente aplicados à resolução de modelos de pequeno porte e simplificados, não suficientes para modelar situações reais.

- sistemas matemáticos heurísticos: estes sistemas são caracterizados por apresentarem algoritmos matemáticos heurísticos, que garantem soluções viáveis e relativamente 'boas', porém não necessariamente ótimas. Geralmente, estes sistemas utilizam algoritmos de busca - desenvolvidos segundo o conceito da inteligência artificial - e métodos baseados em gargalos (predominantemente derivados do conceito da teoria das restrições ${ }^{19}$ ). Os avanços nos recursos computacionais e o desenvolvimento das técnicas tem permitido que estes sistemas sejam disponibilizados comercialmente - muitas vezes na forma de soluções proprietárias fechadas, em que os algoritmos são mantidos em segredo pelos fornecedores, por serem eles os possíveis diferenciais competitivos do produto. O sistemas matemáticos heurísticos podem, em teoria, gerar soluções melhores do que os sistemas baseados em regras de liberação, porém eles são relativamente mais complexos - dado o grau de sofisticação matemática da solução - e em geral exigem um tempo computacional proporcionalmente maior. 
- sistemas especialistas puros: estes sistemas - baseados em conceitos de inteligência artificial - consistem em, através da coleta do conhecimento de especialistas em determinado assunto, transformá-lo em uma série de regras de decisão que, através do denominado "motor de inferência", chegam a uma solução. Os sistemas especialistas puros são, geralmente, soluções ad hoc, com limitada disponibilização em pacotes comerciais; a sua aplicação maior ocorre no desenvolvimento de aplicativos de software específicos - utilizando, para tanto, linguagens tais como PROLOG e Common LISP.

- sistemas apoiados em redes neurais: estes sistemas são um desenvolvimento da inteligência artificial que tentam simular o processo de aprendizado da mente humana. Eles consistem de uma rede formada por diversos níveis e nós que processam a informação e 'acumulam' conhecimento, e são basicamente aplicadas a problemas que denotam uma solução adaptativa e reativa. As pesquisas quanto à aplicação no âmbito da programação da produção são recentes e ainda limitam-se ao campo acadêmico.

b) grau de interação com o usuário:

- sistemas abertos: a principal característica destes sistemas reside na necessidade de interação com o usuário. A metodologia de resolução do problema da programação da produção é de conhecimento do usuário e este pode definir as regras inerentes ao processo de tomada de decisão, bem como alterar as decisões geradas pelo sistema. Desta forma, a responsabilidade pela decisão é do usuário, e o sistema funciona como uma ferramenta de suporte, simulando o efeito das decisões tomadas pelo programador que aborda o problema tentando e errando até encontrar uma solução.

- sistemas fechados: nestes, a responsabilidade pela decisão é do próprio sistema. A interação com o usuário se resume à definição de alguns critérios, como por exemplo, a localização dos gargalos ou a ponderação de objetivos de desempenho que o sistema deve buscar.
Na realidade, esta classificação define dois extremos. Na prática, existem também soluções intermediárias, em que os sistemas fechados mantém a responsabilidade pela decisão, porém permitem um interação maior com o usuário na definição das regras de decisão (podendo ser classificados como 'sistemas semi-fechados'); e os 'sistemas semi-abertos', onde o usuário é responsável pela decisão, porém o sistema limita a escolha da metodologia de decisão.

c) abrangência das decisões no âmbito do planejamento da produção:

- sistemas de apoio ao plano mestre de produção: determinam o plano referente às quantidades e itens de produtos finais a serem produzidos, período a período.

- sistemas de apoio à programação da produção: definem as sequências de ordens a serem executadas nos recursos produtivos em um determinado horizonte de tempo.

- sistemas que executam a gestão dos materiais integrada à capacidade produtiva: gerenciam os estoques de matériasprimas sincronizadamente à capacidade produtiva, determinando as necessidades de aquisição de materiais - em termos de quantidades e do respectivo timing.

\section{A decisão sobre a implantação de um sistema de programação da produção com capacidade finita, considerando as implicaçóes decorrentes, a dificuldade de reverter e o nível de investimento demandado, é uma decisão, por natureza, estratégica.}

21. Para maiores detalhes veja KUSIAK, A. Intelligent Manufacturing Systems. Singapure: Prentice-Hall Inc. 1990 e KERR, R. KnowledgeBased Manufacturing Management. Singapure: Addison-Wesley Publishing Company Inc., 1991. Como exemplos de aplicaçăo, veja BENSANA, E., Bel, G. e DUBOIS, D. OPAL: A Multi-KnowledgeBased System for Industrial JobShop Scheduling. International Journal of Production Research. v. 26 , n. 5 , p. $795-819,1988$ KATHAWALA, Y. e ALLEN, W. R Expert Systems and Job Shop Scheduling. Intemational Joumal of Operations \& Production Management, v. 13, n. 2, p. 2335, 1993.

22. Como exemplos de aplicação veja PHILIPOOM, P. R., REES, L. P. e WIEGMANN, L. Using Neural Networks to Determine InternallySet Due-Date Assignments for Shop Scheduling. Decision Scienses, v. 25, n.. $5 / 6$, p. 825 851, 1994; SIM, S. K., YEO, K. T. e LEE, W. H. An Expert Neural Network System for Dynamic Job Shop Scheduling. International Journal of Production Research, v. 32, n. 8, p. 1759-1773, 1994. 


\section{TABELA 1.}

\begin{tabular}{|c|c|c|}
\hline 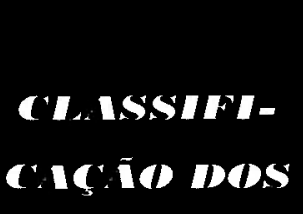 & $\begin{array}{l}\text { - segundo o suporte às } \\
\text { funçōes do planeja- } \\
\text { mento da produção }\end{array}$ & $\begin{array}{l}\text { - baseados em regras de liberação } \\
\text { - matemáticos otimizantes } \\
\text { - matemáticos heurísticos } \\
\text { - sistemas especialistas puros } \\
\text { - apoiados em redes neurais }\end{array}$ \\
\hline 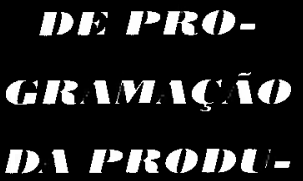 & $\begin{array}{l}\text { - segundo o grau de } \\
\text { interação com o usuá- } \\
\text { rio }\end{array}$ & $\begin{array}{l}\text { - sistemas abertos } \\
\text { - sistemas semi-abertos } \\
\text { - sistemas fechados } \\
\text { - sistemas semi-fechados }\end{array}$ \\
\hline 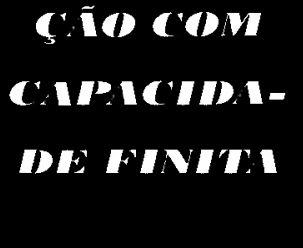 & $\begin{array}{l}\text { - segundo o método de } \\
\text { solução do problema }\end{array}$ & $\begin{array}{l}\text { - plano mestre de produção } \\
\text { - programação da produção } \\
\text { - gestão dos materiais integrada à } \\
\text { capacidade } \\
\text { - controle de produção }\end{array}$ \\
\hline
\end{tabular}

- sistemas que executam o controle da produção: permitem monitorar a realização do plano ou das ordens planejadas.

A classificação dos sistemas de programação da produção com capacidade finita, proposta pelos autores, é apresentada resumidamente na tabela 1.

Algumas observações podem ser feitas a respeito desta classificação:

- os sistemas classificados como abertos permitem que o usuário tenha um domínio maior sobre a solução do problema, o que envolve, por outro lado, a necesidade de um maior conhecimento sobre as regras de resolução do problema da programação da produção. Assim, pode ser necessário um salto de patamar qualitativo em termos do profissional que executa tal tarefa. Os sistemas fechados, além de serem geralmente bastante mais caros, implicam que haja confiança total - ou fé - no algoritmo desenvolvido pelo fabricante, já que nem sempre informações suficientes são disponibilizadas pelos fornecedores;

- os sistemas mais difundidos atualmente são os baseados em regras de liberaçãoe os matemáticos heurísticos. Estes, dada a tecnologia embutida, são geralmente fechados e com soluções proprietárias; por outro lado, os sistemas baseados em regras de liberação, em função da relativa simplicidade de modelagem e da difusão destas regras, são, em geral, abertos.

\section{OS SISTEMAS DE PROGRAMAÇÃO DA PRODU- ÇÃO COM CAPACIDADE FINITA DISPONÍVEIS NO BRASIL}

Um recente trabalho de pesquisa realizado pelos autores identificou os principais sistemas de programação da produção com capacidade finita comercialmente disponíveis no Brasil, até a presente data. As informações foram coletadas a partir de entrevistas com os fornecedores locais destes aplicativos. O resultados são apresentados na tabela 2, bem como as características principais destes, basedas na classificação proposta no item anterior.

As principais observações resultantes deste trabalho foram:

- as informações apresentadas ilustram uma situação correspondente à data da pesquisa (final de 1995). Na realidade, em função da rapidez de desenvolvi- 
mento com que esta área vem passando - na forma de lançamentos de novas versões, novos produtos e até novos conceitos -, estas informações merecem ser revistas com certa freqüência;

- o grau de maturidade e de sofisticação destes sistemas variam consideravelmente, bem como os respectivos preços (dentre os sistemas disponiveis comercialmente no Brasil, foram identificados valores que variaram de sete mil a um milhão e meio de dólares). Em geral, os sistemas com soluções proprietárias fechadas são sensivelmente mais caros;

- uma grande parte destes sistemas encontra-se integrada à banco de dados notadamente Informix, Oracle, Ingress e Progress. Em relação ao sistema operacional, geralmente os sistemas mais sofisticados "rodam" em UNIX e os mais simples em DOS - o ambiente Windows está em fase de desenvolvimento e o sistema OS/2 é menos difundido. Por outro lado, as interfaces entre os sistemas de programação com capacidade finita e os sistemas MRPII disponíveis no mercado encontram-se, ainda, em um estágio inicial;

- nem todos os representantes brasileiros de sistemas fabricados no exterior dominam a tecnologia envolvida no sistema e, notadamente nos casos de sistemas fechados, sabem o que o sistema apresenta por dentro. Neste caso, uma eventual necessidade de customização poderia custar ao usuário tempo e recursos financeiros relativamente altos;

- nem todos os fornecedores e representantes nacionais estão capacitados a dar suporte tanto na análise de adequação da solução a potenciais clientes quanto na própria implantação e apoio técnico ao uso dos aplicativos. Alguns deles dão a impressão de terem pouco conhecimento a respeito daquilo que estão vendendo - em termos de implicações quanto às necessidades estratégicas da empresa e até mesmo a respeito das funcionalidades dos respectivos sistemas -; outros, por outro lado, prometem melhorias que beiram a 'panacéia', promessas que dificilmente poderão ser cumpridas.

Atualmente, existe cerca de uma centena destes sistemas disponíveis comercial- mente em nível mundial. Além dos apresentados na tabela 2 , pode-se citar: OPT (Scheduling Technology Group - Inglaterra), Resonance (Thru-Put Technologies), AFL 1300 Leitstand (Krupp-Atlas Eletronic $\mathrm{GmbH}$ ), FLS-AIX Leitstand (IBM-Alemanha), VPPS Leitstand (Infor GmbH), CMS (Manufacturing Management Systems Inc.), PSA (Stone \& Webster ASDS Inc.), PROVISA (AT\&T Istel), FACT EnSync (Fact Inc.), SHIVA (ShivaSoft Inc.), Job Time Plus (Job Time Systems Inc.), MS/X On Time e TS/X On Time (Tyecin Systems Inc.), TESS (Taylor Manufacturing Systems), AMAPS (D\&B Software Advanced Manufacturing International), WATPASS (Waterloo Engineering Software), MPSwin (Bridgeware Inc.), DSP (Gensym), PST (Johnson Intelligent Systems), OPS (Red Pepper Software Company), PROSPEX (Sira Industrial Systems) e MIT (Strategic Business Solutions Inc.).

Deve-se ainda considerar que existem trabalhos que resultaram em sistemas protótipos, acadêmicos ou em desenvolvimento, entre eles: OPAL (Toulouse), ISIS, OPIS, SONIA, CALLISTO (Digital Equipment Company), PATRIARCH, MERLE, L1 Project (Dortmund), Production Reservation System, Quick Response Planner (Clemson) e LLISS (Cornell University). Com isso pode-se ter uma idéia da ebulição em que se encontra a área de desenvolvimento de sistemas de programação da produção com capacidade finita.

\section{CONCLUSÕES}

A decisão sobre a implantação de um sistema de programação da produção com capacidade finita, considerando as implicações decorrentes, a dificuldade de reverter e o nivel de investimento demandado, é uma decisão, por natureza, estratégica. Desta forma, é fundamental a adequação do sistema às necessidades estratégicas atuais e futuras - da empresa, a consideração dos níveis de investimento e esforços organizacionais exigidos, bem como uma análise criteriosa dos sistemas disponíveis.

Quanto à consideração das necessidades estratégicas, é importante ressaltar que: em primeiro lugar, nem todos os sistemas pro- 


\begin{tabular}{|c|c|c|c|c|c|c|}
\hline & \multirow[b]{2}{*}{ Sottware } & \multirow[b]{2}{*}{ Fabricante } & \multirow[b]{2}{*}{ Origem } & \multicolumn{3}{|c|}{ PRINCIPAIS CARACTERISTICAS } \\
\hline & & & & Método de Solução & $\begin{array}{l}\text { Grau de } \\
\text { Interaçáo }\end{array}$ & Funçōes \\
\hline 1 & $\begin{array}{l}\text { AHP- } \\
\text { Leitstand }\end{array}$ & AHP & Alemanha & regras de liberação & aberto & $\begin{array}{l}\text { - programação da produçáo } \\
\text { - controle da produção }\end{array}$ \\
\hline 2 & $\begin{array}{l}\text { Fl-2 } \\
\text { Leitstand }\end{array}$ & $\begin{array}{l}\text { IDS Prof. } \\
\text { Scheer }\end{array}$ & Alemanha & regras de liberaçāo & aberto & $\begin{array}{l}\text { - programação da produção } \\
\text { - controle da produçāo }\end{array}$ \\
\hline 3 & $\begin{array}{l}\text { FMS } \\
\text { Leitstand }\end{array}$ & Siemens & Alemanha & regras de liberaçáo & aberto & $\begin{array}{l}\text { - programação da produção } \\
\text { - controle da produção }\end{array}$ \\
\hline 4 & $\begin{array}{l}\text { Preactor } \\
200\end{array}$ & $\begin{array}{l}\text { CIMula } \\
\text { tion } \\
\text { Centre }\end{array}$ & Inglaterra & regras de liberação & semi-aberto & - programaçáo da produção \\
\hline 5 & MOOPI & Berclain & Canadá & matemálico heurístico & fechado & $\begin{array}{l}\text { - programação da produção } \\
\text { - gestão dos materiais integrada } \\
\text { - controle de produção }\end{array}$ \\
\hline 6 & Schedulex & Numetrix & Canadá & matemático heuristico & $\begin{array}{l}\text { semi- } \\
\text { fechado }\end{array}$ & $\begin{array}{l}\text { - plano mestre de produção } \\
\text { - programação da produção }\end{array}$ \\
\hline 7. & MRS & \begin{tabular}{|l|} 
Taylor \\
Indl. \\
Software \\
\end{tabular} & Canadá & regras de liberação & aberto & $\begin{array}{l}\text { - programaçáo da produção } \\
\text { - controle da produção }\end{array}$ \\
\hline 8 & Autosched & \begin{tabular}{|l|} 
Auto \\
Simulations
\end{tabular} & EUA & regras de liberaçāo & aberto & - programação da produçāo \\
\hline 9 & Scheduler & Manugistics & EUA & matemático & $\begin{array}{l}\text { semi- } \\
\text { fechado }\end{array}$ & - plano mestre de produção \\
\hline 10 & Rhythm & $\begin{array}{l}\text { i2 } \text { Tech- } \\
\text { nologies }\end{array}$ & EUA & matemático heurístico & $\begin{array}{l}\text { semi- } \\
\text { fechado }\end{array}$ & $\begin{array}{l}\text { - plano mestre de produção } \\
\text { - programação da produção } \\
\text { - gestão dos materiais integrada }\end{array}$ \\
\hline 11 & $\begin{array}{l}\text { The Goal } \\
\text { System }\end{array}$ & $\begin{array}{l}\text { Goal } \\
\text { Systems }\end{array}$ & EUA & matemático heurístico & fechado & $\begin{array}{l}\text { - programação da produçāo } \\
\text { - gestão dos maleriais integrada }\end{array}$ \\
\hline 12 & Factor & $\begin{array}{l}\text { Pristker } \\
\text { Corp. }\end{array}$ & EUA & regras de liberação & aberto & $\begin{array}{l}\text { - programação da produção } \\
\text { - controle da produção }\end{array}$ \\
\hline 13 & Jobbing & INT & Brasil & regras de liberação & aberto & - programação da produção \\
\hline 14 & $\begin{array}{l}\text { Prodira } \\
1000\end{array}$ & \begin{tabular}{l|} 
Coprodin \\
Eletrônica
\end{tabular} & Brasil & regras de liberação & $\begin{array}{l}\text { semi- } \\
\text { aberto }\end{array}$ & - programação da produçāo \\
\hline
\end{tabular}


dutivos demandam uma solução desta natureza para a gestão da sua capacidade; em segundo lugar, o escopo das decisões apoiadas por estes sistemas varia - desta forma, cabe verificar se estas estão alinhadas às decisões fundamentais à gestão do sistema de PPCP da empresa no que se refere à geração do plano mestre de produção, à programação da produção, à gestão dos materiais integrada à capacidade e ao controle da produção; e, finalizando, é primordial verificar se o sistema escolhido comporta as características particulares da empresa que são relevantes à decisão apoiada.

Um importante fator de êxito, uma vez escolhido o sistema correto, é a metodologia de implantação. Esta deve considerar o envolvimento dos recursos humanos e o treinamento destes na etapas corretas das fases de implantação; estas etapas, por outro lado, devem ser gerenciadas dentro do conceito de gestão de projetos.

Por outro lado, é também importante avaliar o grau de capacitação e a postura profissional do fornecedor ou representante comercial. Cabe lembrar que, uma vez implantado o sistema, pode-se criar uma dependência de seu fornecedor, tanto em relação à assistência técnica quanto à tecnologia adquirida. Esta última é particularmente importante nos sistemas que apresentam soluções proprietárias fechadas, uma vez que o sistema assume uma parcela de responsabilidade pela decisão dentro do sistema de PPCP.

É importante reforçar que um sistema de programação da produção com capacidade finita não deve ser gerenciado de maneira isolada, ou seja, não integrado às demais dimensões temporais do planejamento, bem como às demais funçóes da empresa. Neste contexto, estes sistemas podem assumir uma função de complementação em relação aos sistemas do tipo MRPII, substituindo o módulo de controle de fabricação quando a complexidade do sistema produtivo demandar gestão de capacidade finita.

Os sistemas de programação da produção com capacidade finita estão apoiados em informações - tanto quanto a modelagem do sistema produtivo, quanto dos parâmetros para a tomada de decisões e da

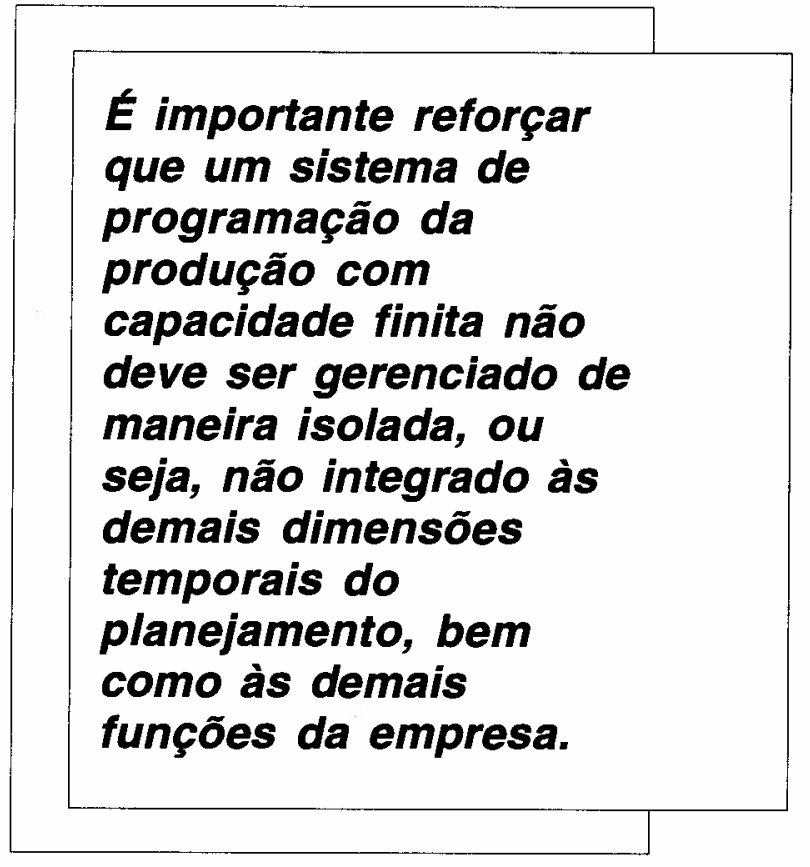

situação presente da fábrica. Isto significa que a qualidade da decisão resultante é particularmente dependente da confiabilidade, integridade e aderência destas informações à realidade modelada.

Um fator importante diz respeito à complexidade do sistema produtivo. Pode-se utilizar o sistema de PPCP para buscar atingir um melhor desempenho de um sistema desnecessariamente complexo - por exemplo, em fábricas não focalizadas e em processos não-estáveis. Neste caso pode ser aconselhável diminuir a complexidade deste antes da implantação de um sistema de programação da produção com capacidade finita. Também pode ser conveniente utilizar sistemas de PPCP híbridos ${ }^{24}$ - ou seja, sistemas com características particulares por linhas de produtos ou por 'minifábricas', por exemplo. Este caso implica que um sistema de programação da produção com capacidade finita não necessita ser necessariamente implantado na fábrica toda. Isto pode ser o caso de empresas com produção altamente repetitiva (para a qual se decida utilizar MRPII ou princípios JIT) com, por exemplo, ferramentarias complexas (por ex.: fabricantes de eletrodomésticos). Neste caso, possivelmente apenas a ferramentaria necessitaria de uma solução de programação da produção com capacidade finita.
23. No caso de processos não estáveis, essa afirmação é reforçada por meio de um estudo realizado por MATSUURA, $H$., TSUBONE, H. E KATAOKA, K. Comparison between Simple Infinite Loading and Loading Considering a Workload Status under Uncertainty in Job Operation Times. International Joumal of Production Economics, v. $40, p .45-55,1995$ que, comparando as técnicas de programação da produção com capacidade infinita e finita, demonstrou claramente a superionidade desta última; porém a vantagem da programação com capacidade finita foi reduzida quando aplicada em condiçóes em que os tempos de operaçáo reais apresentavam grandes variações em relação aos previstos. Ainda, MELNYK, S. A., DENZLER, D. R. e FREDENDALL, L. Variance Control Vs. Dispatching Efficiency. Production and Inventory Management Journal, Third Quarter, 1992, comprovam que os objetivos de desempenho do sistema de PPCP obtidos por simulaçáo de diferentes regras de liberação são fortemente afetados pelas variabilidades do sistema produtivo.

24. Para maiores detalhes sobre sistemas de PPCP híbridos veja BHATTACHARYA, A. K. e COLEMAN, J. L. Linking Manufacturing Strategy to the Design of a Customized Hybrid Production Control System. Computer Integrated Manufactuning Systems, v. 7, n. 2, p. 134-141, 1994. 